\title{
Ferromagnetism in the Hubbard model with an infinite-range hopping.
}

\author{
P. Pieri \\ Dipartimento di Fisica, INFM and INFN, Università di Bologna, \\ Via Irnerio 46, I-40126, Bologna, Italy. \\ (Fax: +39 51 6305153, Tel: +39 51 6305110, E-mail: pieri@bologna.infn.it)
}

\begin{abstract}
We prove, as recently conjectured, that the ground state of the Hubbard Hamiltonian with an infinite-range hopping, when the number of electrons $N_{e}=N+1$ ( $N$ being the number of sites), is ferromagnetic fully polarized.
\end{abstract}

PACS: 71.10; 75.10L.

Despite its long history, the problem of itinerant ferromagnetism is still, to many regards, an open question. The Hubbard model was considered from the beginning as a good model to investigate this intriguing problem [四]. However, rigorous examples of ferromagnetism in the Hubbard model have been shown only in some rather peculiar situations: Nagaoka ferromagnetism for infinite Hubbard repulsion $U$ and one hole in a half-filled band [2] (see [3] for a very elegant proof of Nagaoka's theorem), Lieb ferrimagnetism for halffilled bipartite lattices with sublattices containing a different number of sites [四] and the flat (or nearly-flat) band of Mielke [5] and Tasaki [6]. Besides these rigorous results, some recent numerical and analytical investigations seem to indicate the existence of a fully polarized ferromagnetic ground state in the one dimensional Hubbard model with nearest and next-nearest-neighbour hopping [7, 8]. In the presence of so few results, it is clear that every new example of ferromagnetism in a Hubbard model is of great interest.

Very recently some conjectures about the ground state of the Hubbard model 
with an infinite-range hopping appeared in literature [9, 10] (see eq. (四) below for the definition of the Hamiltonian, and refs. [11, 12 for some previous works on this model). Among these, one is relevant to the discussion of itinerant ferromagnetism. According to ref. [10], when the filling corresponds to just one electron more than half-filling, the ground state of the model of Eq. (11) below should be a Nagaoka ferromagnetic state for every value of $U>0$. Now, it is easy to check that the model (11), when $U=\infty$ and after a particle-hole transformation, satisfies the conditions for the applicability of Tasaki's generalization of Nagaoka's theorem. Interestingly, the conjecture of ref. [10] extends, for this specific model, the region of Nagaoka ferromagnetism from $U=\infty$ down to vanishingly small $U$. In this work we shall give a rigorous proof for this conjecture.

We consider now the Hubbard Hamiltonian:

$$
H=-t \sum_{\sigma, 1 \leq i \neq j \leq N} c_{i \sigma}^{\dagger} c_{j \sigma}+U \sum_{i=1}^{N} n_{i \downarrow} n_{i \uparrow},
$$

The infinite-range and costant hopping in the Hamiltonian (1), makes the system invariant under the action of the permutation group $S_{N}$ ( $N$ being the number of lattice sites). The Hamiltonian has therefore a block-diagonal form, with the blocks classified according to the irreducible representation of $S_{N}$. The symmetry properties under $S_{N}$ and the total spin of the ground state were studied in refs. [9, 10], on small size systems, by exact diagonalization of the Hamiltonian (1). These symmetry properties and the value of the ground state's total spin were then supposed to hold also for systems of arbitrary size. In particular, while below half-filling the ground state should be a spin singlet, at the filling $N_{e}=N+1$ the ground state should be fully polarized: $S=(N-1) / 2$ (here $S$ labels as usual the eigenvalues $S(S+1)$ of the total spin operator $\left.S^{2}\right)$. Finally, for filling $N_{e}>N+1$, the ground state should become completely degenerate with respect to $S$. Hence, only when $N_{e}=N+1$ the ground state should be ferromagnetic (and fully polarized). To find out the ground state of $H$ when $N_{e}=N+1$ we now need to state a general theorem proven by Mielke on flat-band ferromagnetism [5].

Let us assume that the hopping matrix $T=\left(t_{i j}\right)_{i, j=1, \ldots, N}$ of a Hubbard Hamiltonian $H$ has a lowest eigenvalue $\lambda_{0}$ with degeneracy $N_{d}$. Let $f_{i \sigma}^{\dagger}$ $\left(i=1, \ldots, N_{d}\right)$ be the creation operator of one electron with spin $\sigma$ in one of 
the $N_{d}$ degenerate states with eigenvalue $\lambda_{0}$. Let us define:

$$
|\psi\rangle=\prod_{i=1}^{N_{d}} f_{i \uparrow}^{\dagger}|0\rangle
$$

The state $|\psi\rangle$ has total spin $S=\frac{N_{d}}{2}$ and $S_{z}=\frac{N_{d}}{2}$ and is therefore fully polarized. We introduce now the matrix $\rho$, whose elements are defined by:

$$
\rho_{j j^{\prime}}=\left\langle\psi\left|c_{j \uparrow}^{\dagger} c_{j^{\prime} \uparrow}\right| \psi\right\rangle
$$

where $j, j^{\prime}=1, \ldots, N$ label the Wannier states. Let $L$ be the subset that contains the sites corresponding to the nonvanishing lines (or columns) of $\rho$. Theorem: (Mielke) If and only if the matrix $\rho$ restricted to $L$ is irreducible, then for every $U>0,|\psi\rangle$ is the unique ground state of $H$ with $N_{e}=N_{d}$ electrons, up to the trivial $S_{z}$ degeneracy.

We recall that a square matrix $A$ is called reducible if there is a permutation of the basis' vectors that puts it into the form:

$$
\widetilde{A}=\left(\begin{array}{cc}
B & 0 \\
C & D
\end{array}\right)
$$

where $B$ and $D$ are square matrices (of course when $A$ is hermitian $C=0$ ). Otherwise $A$ is called irreducible.

Let's go back now to the Hamiltonian (11) that defines the Hubbard model with infinite-range hopping. We first apply a particle-hole transformation to $H$. The transformation, which is defined by the unitary operator $I=$ $\prod_{i, \sigma}\left(c_{i \sigma}^{\dagger}+c_{i \sigma}\right)$, transforms some of the Fock space operators as follows:

$$
\begin{aligned}
& I c_{i \sigma} I^{\dagger}=c_{i \sigma}^{\dagger} \\
& \tilde{H} \equiv I H I^{\dagger}=t \sum_{\sigma, 1 \leq i \neq j \leq N} c_{i \sigma}^{\dagger} c_{j \sigma}+U \sum_{i=1}^{N} n_{i \downarrow} n_{i \uparrow}+U\left(N-\hat{N}_{e}\right) \\
& I \hat{N}_{e} I^{\dagger}=2 N-\hat{N}_{e} \\
& I \vec{S} I^{\dagger}=-\vec{S}
\end{aligned}
$$

where $\hat{N}_{e}$ is the particle number operator. From these relations it follows that if $|\psi\rangle$ is the ground state of the Hamiltonian $\tilde{H}$ in the subspace with $N_{e}=N-1$ particles, then $I^{\dagger}|\psi\rangle$ is the ground state of $H$ in the subspace 
with $N_{e}=N+1$ particles. Moreover, from the relation (8) it follows that if $|\psi\rangle$ is an eigenstate of $S^{2}$ with eigenvalue $S$, then $I^{\dagger}|\psi\rangle$ is also an eigenstate of $S^{2}$ with the same eigenvalue. We can then look for the spin of the ground state of $\tilde{H}$ in the subspace with $N_{e}=N-1$ to prove the conjecture of ref. 10 about the original Hamiltonian $H$.

The one-body part of $\tilde{H}$ is diagonalized as usual by introducing the Fouriertransformed operators: $c_{k \sigma}=\frac{1}{\sqrt{N}} \sum_{j=1}^{N} e^{i k j} c_{j \sigma}$ with $k=0, \frac{2 \pi}{N}, \ldots, \frac{2 \pi(N-1)}{N}$. The eigenvalues of the hopping matrix $T$ are given by $E_{k}=t\left(N \delta_{k, 0}-1\right)$. The lowest eigenvalue of $T$ is then $-t$ and has degeneracy $N-1$. Hence, when the number of particles $N_{e}=N-1$, one of the conditions for the validity of Mielke's theorem is satisfied $\left(N_{e}=N_{d}\right)$. The other condition to be satisfied is the irreducibility of the matrix $\rho$ in the subspace $L$ defined above. In the present case the state $|\psi\rangle$ of formula (2) is given by:

$$
|\psi\rangle=\prod_{k \neq 0} c_{k \uparrow}^{\dagger}|0\rangle
$$

The matrix $\rho$ is then given by:

$$
\begin{aligned}
\rho_{j j^{\prime}} & =\left\langle\psi\left|c_{j \uparrow}^{\dagger} c_{j^{\prime} \uparrow}\right| \psi\right\rangle=\frac{1}{N} \sum_{k, k^{\prime}}\left\langle\psi\left|c_{k \uparrow}^{\dagger} c_{k^{\prime} \uparrow}\right| \psi\right\rangle e^{i\left(k j-k^{\prime} j^{\prime}\right)} \\
& =\frac{1}{N} \sum_{k, k^{\prime}} \delta_{k, k^{\prime}}\left(1-\delta_{k, 0}\right) e^{i\left(k j-k^{\prime} j^{\prime}\right)}=\delta_{j j^{\prime}}-1 / N
\end{aligned}
$$

Since the matrix elements $\rho_{j j^{\prime}}$ are all different from zero, the subset $L$ we defined above coincides with the whole lattice. The matrix $\rho$ is then irreducible since, as it follows at once from the definition, a necessary condition for a matrix to be reducible is to have at least one element equal to zero. We conclude then that, owing to Mielke's theorem, the ground state of $\tilde{H}$ when $N_{e}=N-1$, is unique (up to the $S_{z}$ degeneracy) and has total spin $S=\frac{N-1}{2}$. We have therefore that, for the original Hamiltonian $H$, the ground state in the subspace with $N_{e}=N+1$ is unique and has total spin $S=\frac{N-1}{2}$, which is the conjecture we wanted to prove.

Wang has recently given some variational arguments in favour of this conjecuture [13]. His arguments are, however, not conclusive since he considered the stability of the fully polarized state with respect to a single spin-flip only. Moreover, since it's not obvious, it should be explicitly shown that a linear combination of the lowest energy states of the hopping part, in the subspace 
with $S=\frac{N-3}{2}$ has always a nonzero probability to have two electrons on the same site, as it is argued in [13].

We would like now to make some comments about Nagaoka's theorem and its implications for the model we have considered. As we said before, it's easy to check that the Hamiltonian $\tilde{H}$, when $U=\infty$ and $N_{e}=N-1$ satisfies the conditions for Tasaki's extension of Nagaoka's theorem. The hoppings $t_{i j}$ are $\geq 0$ as required by Tasaki in [3] and the more delicate connectivity condition also stated by Tasaki [3] is trivially satisfied since, in the present case, the hopping matrix connects directly all the lattice's sites. After Nagaoka's work, a central question in the theory of itinerant ferromagnetism has been: "Does Nagaoka's theorem hold also for a finite $U$ and a small but not null hole density?" [14]. In this paper we have proven that for the Hamiltonian $\tilde{H}$, when $N_{e}=N-1$, Nagaoka's theorem can be extended up to vanishingly small $U$. We have no rigorous arguments to prove the conjectures of refs. [9, 10] for other filling factors, but we believe that our proof for the specific case $N_{e}=N-1\left(N_{e}=N+1\right.$ in the original Hamiltonian) supports also the conjectures for other fillings. In fact the conjectures of refs. [9, 10] are based on some symmetry arguments that seem to be independent of the filling. If we believe then to these conjectures, we can conclude that, when $N_{e}<N-1$ the ground state of $\tilde{H}$ is completely degenerate with respect to the total spin $S$. The Hamiltonian $\tilde{H}$ provides therefore a rather bizarre example where, with one extra hole Nagaoka's ferromagnetism holds up to vanishingly small $U$, while with more than one hole it disappears completely. Thus, this pathological example tells us, one time more, that out of the strict limit $U=\infty$ and one hole, every behaviour is possible and no definite conclusions can be drawn from Nagaoka's theorem.

I wish to thank D. Baeriswyl for having introduced me to the fascinating subject of itinerant ferromagnetism. I am also grateful to G. Morandi, E. Ercolessi and M. Roncaglia for helpful and stimulating discussions.

\section{References}

[1] Hubbard, J.: Proc. Roy. Soc. London A 276, 238 (1963); Kanamori, J.: Progr. Theor. Phys. 30, 275 (1963).

[2] Nagaoka, Y.: Phys. Rev. 147, 392 (1966). 
[3] Tasaki, H.: Phys. Rev. B 40, 9192 (1989).

[4] Lieb, E.: Phys. Rev. Lett. 62, 1201 (1989).

[5] Mielke, A.: Phys. Lett. A 174, 443 (1993).

[6] Tasaki, H.: Phys. Rev. Lett. 69, 1608 (1992); Mielke, A. and Tasaki, H.: Comm. Math. Phys. 158, 341 (1993); Tasaki, H.: Phys. Rev. Lett. 75, 4678 (1995).

[7] Müller-Hartmann, E.: J. Low. Temp. Phys. 99, 349 (1995).

[8] Pieri, P., Daul, S., Baeriswyl, D., Dzierzawa, M., and Fazekas, P.: condmat/9603163.

[9] Salerno, M.: Z. Phys. B 99, 469 (1996).

[10] Salerno, M.: Eolv-int/9603008, to appear in Z. Phys. B (1996).

[11] van Dongen, P. and Vollhardt, D.: Phys. Rev. B 40, 7252 (1989).

[12] Verges, J. A., Guinea, F., Galan, J., van Dongen, P., Chippe, G. and Louis, E.: Phys. Rev. B 49, 15400 (1994).

[13] Wang, D. F., cond-mat/9604121.

[14] An account of the efforts made in this direction (and much more) can be found in: Lieb, E. in The Hubbard Model, its Physics and Mathematical Physics, edited by Baeriswyl, D. et al., NATO ASI Series (Plenum Press, New York, 1995), pp. 1-19, and references therein. 